the cohort (SMR 2.25, 43 observed), which however had an inverse relation to blood lead level (SMRS 11.1, 3.2, 0.9. 0.8 by increasing category). Numbers were small for other causes of interest (stomach cancer $\mathrm{n}=23$, kidney cancer $\mathrm{n}=28$, brain cancer $\mathrm{n}=31)$. SMRs for stroke were low $(0.59,0.57,0.81$. 0.87 ) but showed a borderline significant increasing trend in Poisson regression $(\mathrm{p}=-0.08)$.

Conclusion We studied a large cohort with documented lead exposure. Results are not conclusive but suggest an association between lung cancer and lead exposure. Data are limited by a lack of work history, a limited blood leads per person, and relatively short latency.

\section{CHOLANGIOCARCINOMA AMONG WORKERS IN THE PRINTING INDUSTRY: USING THE NORDIC OCCUPATIONAL CANCER DATABASE TO ELUCIDATE A CLUSTER REPORT FROM JAPAN}

1. I Vlaanderen, ${ }^{1}$ Straif, ${ }^{2}$ Martinsen, ${ }^{3}$ Kauppinen, ${ }^{4}$ Pukkala, ${ }^{5}$ Sparen, ${ }^{6}$ Tryggvadottic, ${ }^{7}$ Weiderpass, ${ }^{2}$ Kjaerheim. ${ }^{1}$ IARC, Lyon, France; ${ }^{2}$ Cancer registry of Norway, Oslo, Norway; ${ }^{3}$ Finnish Institute of Occupational Health, Helsinki, Finland; ${ }^{4}$ School of Public Health, University of Tampere, Tampere, Finland; ${ }^{5}$ Department of Medical Epidemiology and Biostatistics, Karolinska Institute, Stockholm, Sweden; ${ }^{6}$ Faculty of Medicine, University of Iceland, Reykjavik, Iceland; ' Folkhalsan Researchcenter, Samfundet Folkhasan, Helsinki, Finland

\subsection{6/oemed-2013-101717.279}

Objectives A cluster of 11 cases of cholangiocarcinoma (CC) was recently observed in a small Japanese printing firm. To explore whether the identified cluster is indicative for an elevated risk of CC among workers in the printing industry at large we explored the risk of liver cancer, gall bladder cancer and CC among individuals employed in the printing industry in four Nordic countries (Finland, Iceland, Norway, and Sweden) over a period of 45 years. We used data from the Nordic Occupational Cancer (NOCCA) cohort.

Methods The cohort was set-up by linking occupational information from censuses to national cancer registry data utilising personal identity codes in use in all Nordic countries. We calculated standardised incidence ratios (SIRs) for men and women working in the printing industry, stratified by occupational category (typographers, printers, lithographers, bookbinders).

Results Among men we observed elevated SIRs for cancer of the liver (1.35, 95\% CI: 1.14-1.60; 142 cases), specifically intrahepatic CC $(2.34,95 \%$ CI: $1.45-3.57 ; 21$ cases). SIRs for liver cancer were especially elevated among printers and lithographers, and SIRs for intrahepatic CC among typographers and printers. SIRs for cancer of the gall bladder or extrahepatic CC were not elevated. SIRs for women followed a similar pattern, but the number of cases was low.

Conclusions The NOCCA cohort has proven useful for an instant investigation following-up a report of a cancer cluster. Our study supports the notion that the finding of excess CC risk among workers in a small Japanese printing firm possibly extends beyond that specific firm and country. Further studies should focus on the specific exposures that occur in the printing industry.

\section{Session: 11. Neurological outcomes}

280 A LONGITUDINAL STUDY OF NEUROPSYCHOLOGICAL FUNCTION IN YOUNG MALE DIVERS
R B P Bast-Pettersen, Skare, Skogstad. National Institute of Occupational Health, Oslo, Norway

\subsection{6/oemed-2013-101717.280}

Background and Objective Exposure to compressed-air diving may affect the nervous system. The aim of the present study was to prospectively assess possible nervous system effects from diving. A further aim was to study the effect of age on neuropsychological function in healthy young men.

Methods We obtained baseline observations of 50 young men while they were trainees at a professional diving school and retested them after six $(\mathrm{N}=43)$ and twelve $(\mathrm{N}=37)$ years. Average age at the first test examination was 25 years. The subjects underwent an interview focusing on education, life style habits, accidents and illnesses, and they answered a neuropsychiatric questionnaire. Number of dives, years of diving and being a professional diver or not was recorded. They were tested with a comprehensive neuropsychological test battery comprising tests for problem solving, attention/working memory, speed of information processing, motor skills and reaction times.

Results At the end of the follow-up, 16 divers reported to be professional divers (mostly working part-time as diver). Mean number of cumulated dives was 1250 among the professional divers and 400 among the non-professional divers. Diving exposure was not found to be associated with impaired neuropsychological test results during the 12 year follow-up. There was a tendency to an increase in number of self-reported neuropsychiatric symptoms among the oldest divers, but diving activity was not related to an increase in number of symptoms. This group of young men tested three times, had on average similar, and for several tests, almost identical results during this 12 year follow-up.

Conclusion Diving exposure did not seem to be associated with impaired neuropsychological test results in this 12 -year longitudinal study.

\section{OCCUPATIONS AND PARKINSON'S DISEASE IN A POPULATION-BASED CASE-CONTROL STUDY}

${ }^{1}$ M A Harris, ${ }^{2}$ Marion, ${ }^{2}$ Tsui, ${ }^{2}$ Shen, ${ }^{2}$ Teschke. ${ }^{1}$ Ryerson University, Toronto, Canada; ${ }^{2}$ University of British Columbia, Vancouver, Canada

10.1136/oemed-2013-101717.281

Objectives Despite mounting evidence on epidemiological risk factors, Parkinson's disease (PD) incidence remains largely unexplained. The purpose of this study was to test associations between occupations and PD to complement analyses of occupational exposures.

Methods We used a population-based case-control design in British Columbia, Canada, including 403 cases (users of antiparkinsonian medication) and 405 controls (from the provincial health insurance register, frequency matched on age and sex). Job histories were collected using structured questionnaires querying all jobs held since age 16. Odds ratios were calculated for associations between PD and occupational categories using unconditional logistic regression, adjusting for age, sex, and smoking.

Results Significantly elevated risks were found for social science, law and library jobs (OR 1.82; 95\% CI 1.01 - 3.29); and farming and horticulture jobs (OR 2.03; 95\% CI 1.10 - 3.74). Nonsignificantly elevated risks were found for gas station jobs 